\title{
EVALUACIÓN DE LA MACROFAUNA DEL SUELO EN ROTACIONES CULTIVOS-PASTURAS CON LABOREO CONVENCIONAL
}

\author{
María Stella Zerbino \\ INIA La Estanzuela, Colonia 70000, Uruguay. E-mail: szerbino@inia.org.uy
}

Zerbino, M. S. 2010. Evaluación de la macrofauna del suelo en rotaciones cultivos-pasturas con laboreo convencional. Acta Zoológica Mexicana (n.s.), Número Especial 2: 189-202.

RESUMEN. Los invertebrados que habitan el suelo son actores importantes en los procesos edáficos. La fauna edáfica comprende a organismos con tamaños y estrategias adaptativas diferentes. Los de mayor tamaño, constituyen la macrofauna (ancho del cuerpo mayor a $2 \mathrm{~mm}$ ), que se destaca porque directa o indirectamente afectan las propiedades del suelo. Las comunidades presentes son la consecuencia de las prácticas de manejo de suelo que se realizan, por lo que tienen gran potencial de uso como indicadores. Este trabajo tuvo como objetivo evaluar el efecto de diferentes sistemas de rotación cultivos-pasturas sobre las comunidades de la macrofauna del suelo. Los tratamientos evaluados fueron: agricultura continua $\sin (\mathrm{S} 1)$ y con fertilización (S2), rotación agricultura 33\% - pradera $66 \%(\mathrm{~S} 4)$, rotación agricultura $50 \%$ - pradera $50 \%$ (S5), y rotación agricultura $66 \%$ - pradera $33 \%$ (S7). El número de individuos varió de acuerdo a la intensidad de los usos del suelo evaluados. Los tratamientos S1 y S4 tuvieron respectivamente, las menores y mayores densidades poblacionales de la mayoría de los taxones. El análisis de Co-Inercia entre las propiedades edáficas y la densidad fue significativo. El primer eje ordenó los usos del suelo de acuerdo a su intensidad. Los taxones Oligochaeta y Coleoptera adultos estuvieron asociados a S4, sistema con mayor cantidad de carbono orgánico y nitrógeno total. La evaluación de la macrofauna del suelo conjuntamente con las propiedades del mismo, es una herramienta útil para evaluar la sustentabilidad ambiental de los distintos usos del suelo.

Palabras clave: Fauna del suelo, lombrices de tierra, rotaciones, labranza convencional.

Zerbino, M. S. 2010. Evaluation of soil macrofauna in crop-pasture rotations with conventional tillage. Acta Zoológica Mexicana (n.s.), Número Especial 2: 189-202.

SUMMARY. Soil-inhabiting invertebrates play an important role in soil processes. Soil fauna include organisms of diverse sizes and adaptive strategies. Macrofauna, large organisms with body width greater than $2 \mathrm{~mm}$, influence soil properties, both directly and indirectly. Their communities are influenced by soil management and thus, have a great potential as bioindicators. The objective of this study was to evaluate the effect of different crop-pasture rotations on soil macrofauna. Treatments included continuous agriculture without (S1) and with (S2) fertilization, 33\% crop - 66\% pasture rotation (S4), 50\% croppasture rotation (S5), and $66 \%$ crop - 33\% pasture rotation (S7). The number of organisms collected varied with land use intensity; treatments S1 and S4 had the lowest and highest population densities for most taxa, respectively. The Co-Inertia analysis between soil properties and population density was

Recibido: 16/05/2008; aceptado: 08/01/2010. 
significant. The first axis ordered land uses in relation to its intensity. Oligochaeta and Coleoptera adults were associated with S4, the system with highest organic carbon and total nitrogen contents. The evaluation of soil macrofauna together with soil properties is a useful tool to assess land use sustainability.

Key words: Soil fauna, earthworms, rotations, conventional tillage

\section{INTRODUCCIÓN}

El suelo es un recurso natural esencial que proporciona servicios ecosistémicos en los cuales algunos invertebrados están involucrados (Lavelle et al. 2006). En el perfil del suelo se alojan comunidades diversas de animales que están organizadas en niveles de acuerdo al tamaño y cada componente tiene un rol determinado en su nicho específico, que difícilmente puede ser reemplazado por otros presentes en el sistema. Incluye una variedad de organismos con tamaños y estrategias adaptativas muy diferentes, especialmente en cuanto a la movilidad y modo de alimentación, lo que determina como influyen en los procesos del suelo (Linden et al. 1994, Lavelle 2002). Interviene en los procesos edáficos de dos maneras, directamente por la modificación física de los residuos y del suelo e indirectamente a través de las interacciones con la comunidad microbiana (González et al. 2001). Los invertebrados de mayor tamaño (ancho del cuerpo mayor a $2 \mathrm{~mm}$ ), constituyen la macrofauna, que se destaca porque su actividad tiene efectos en la fertilidad y estructura del suelo, en la infiltración y determina la actividad de los organismos más pequeños (Curry 1987b, Curry \& Good, 1992).

Desde el momento que un sistema natural es modificado para desarrollar actividades agrícolas, los mayores cambios ocurren en las propiedades del suelo y en su biota asociada. Las actividades humanas a través de las distintas prácticas de manejo y tecnologías aplicadas ejercen importantes efectos en los determinantes de la biota del suelo y sobre ella misma, lo que afecta la composición de las comunidades y su nivel de actividad (Lavelle et al. 1993). Las comunidades presentes van a estar determinadas por la intensidad del cambio inducido respecto al ecosistema natural y por la habilidad de los organismos para adaptarse a esos cambios (Brown et al. 2001).

La macrofauna responde a las prácticas de manejo del suelo (secuencia de cultivos, método de preparación del suelo, ingreso de materia orgánica fresca, etc.) en escalas de tiempo de meses o años, como resultado de las perturbaciones físicas que se producen, de la distribución de los residuos y de las comunidades de plantas presentes (Lavelle \& Spain, 2001, Wardle 1995). Esto facilita su potencial como indicadores biológicos.

Para la evaluación de la sustentabilidad ambiental de las innovaciones tecnológicas es necesario disponer de indicadores que permitan alertar sobre el impacto que tendrán las mismas en el largo plazo. Históricamente, la evaluación de los sistemas de producción se centró en los aspectos físicos, mientras que la valoración de los procesos biológicos ha sido escasamente contemplada. 
Las especies cuya presencia o abundancia reflejan alguna característica del hábitat dentro del cual se encuentran, pueden ser consideradas como bioindicadoras (McGeoch \& Chown 1998). La elección de un indicador debe ser realizada para situaciones locales específicas (Elliot 1997) y los indicadores básicos deben ser útiles en un rango de situaciones ecológicas y socioeconómicas (Doran \& Safley 1997)

El presente trabajo tuvo como objetivo determinar el efecto de diferentes sistemas de rotación cultivos-pasturas sobre la estructura de las comunidades de la macrofauna del suelo y explorar las relaciones con las propiedades químicas y físicas del mismo. Por otra parte buscó determinar los grupos taxonómicos asociados a los usos del suelo evaluados.

\section{Descripción del experimento}

\section{MATERIAL Y MÉTODOS}

El muestreo se realizó en un experimento ubicado en la Estación Experimental La Estanzuela (Depto. de Colonia, Uruguay) del Instituto Nacional de Investigación Agropecuaria, instalado en 1963 sobre un suelo de tipo Brunosol Eutrico (franco, arcillo, limoso).

En este experimento se evalúan diferentes sistemas de rotaciones cultivos-pasturas en laboreo convencional y sin pastoreo, que contrastan principalmente en la relación de períodos bajo pastura y bajo agricultura de granos. Las parcelas tienen un tamaño de $25 \mathrm{~m}$ de ancho por $200 \mathrm{~m}$ de largo, instaladas a lo largo de la pendiente. Cada tratamiento cuenta con tres repeticiones en bloques al azar.

Los tratamientos evaluados fueron: S1 - agricultura continua sin fertilización (sorgo - cebada - girasol $2^{\mathrm{a}}$ - trigo); S2 - agricultura continua con fertilización (secuencia idéntica a S1); S4 -agricultura (trigo y cebada) en rotación con pasturas de leguminosas y gramíneas, con $33 \%$ del tiempo en cultivos y $66 \%$ con trébol rojo y pradera mezcla de leguminosas y gramíneas; S5 -agricultura (sorgo, cebada, girasol $2^{\mathrm{a}}$, trigo) en rotación con pasturas mezcla de leguminosas y gramíneas, con 50\% del tiempo en cultivos y la otra mitad con pradera mezcla de leguminosas y gramíneas; y S7 - agricultura (cebada, girasol $2^{\mathrm{a}}$, trigo) en rotación con trébol rojo, con $66 \%$ del tiempo en cultivos y el resto con pasturas (Cuadro I).

\section{Mediciones de campo}

El método de muestreo fue similar al recomendado por el Tropical Soil Biology and Fertility Programme (TSBF) (Anderson \& Ingram 1993). La unidad básica de muestreo fue de $25 \mathrm{~cm}$ de lado por $20 \mathrm{~cm}$ de profundidad. En cada repetición se realizaron 15 unidades básicas de muestreo. Los muestreos se llevaron a cabo en los meses de septiembre (primavera, 2004) y abril (otoño, 2005), porque es la época de mayor abundancia de la fauna del suelo. 


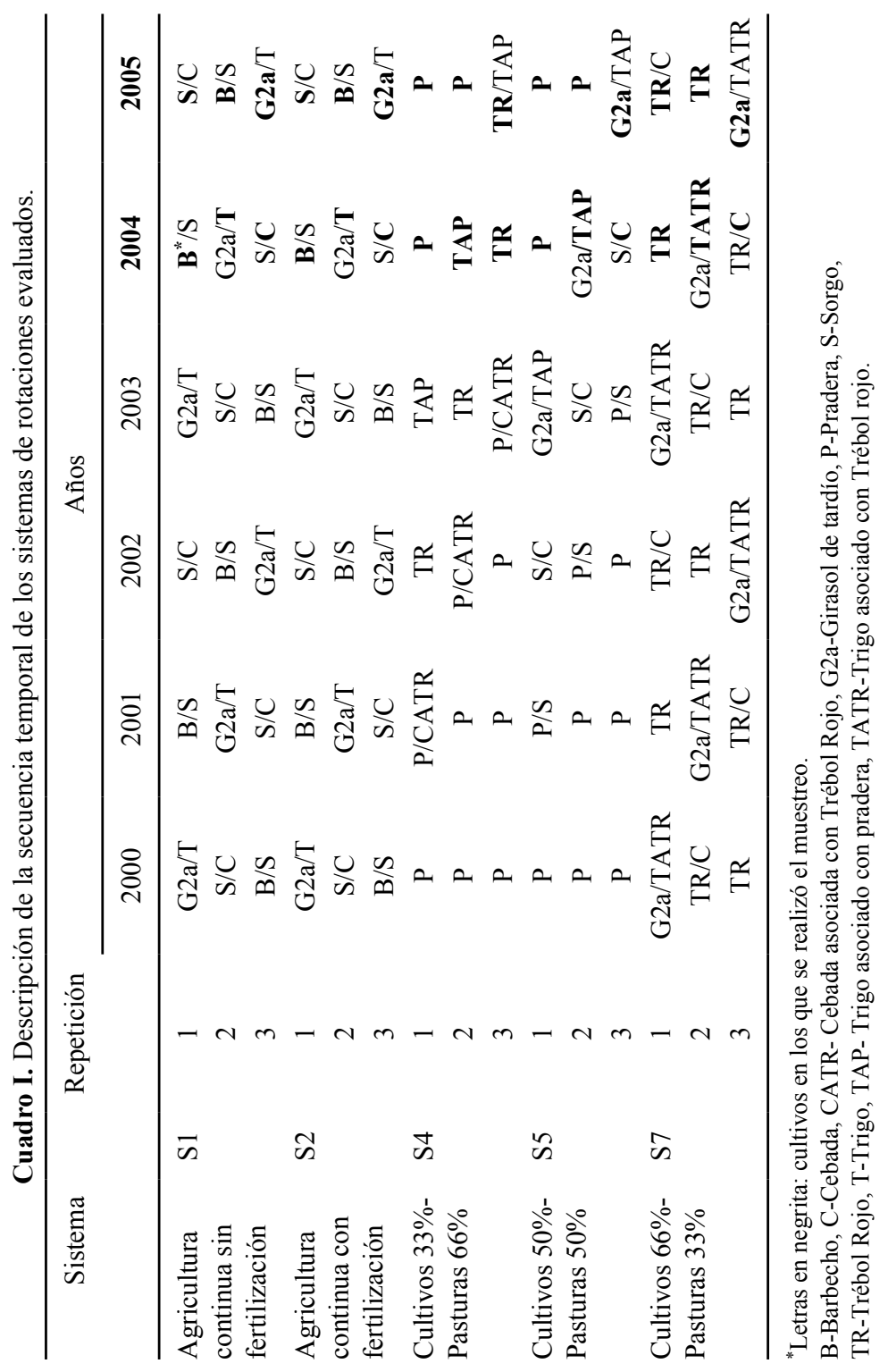


Los artrópodos colectados en cada unidad de muestreo fueron conservados en alcohol 70\% y las lombrices en formol 4\%. En el laboratorio fueron separados de acuerdo a su morfología y clasificados en distintos grupos taxonómicos (Cuadro II).

En cada estación de muestreo se recogieron tres muestras de los $10 \mathrm{~cm}$ superiores del perfil del suelo, para obtener una muestra compuesta de la parcela de 45 submuestras. Las determinaciones realizadas fueron: textura (Bouyoucos), Carbono orgánico (C.Org.) (Tinsley), Nitrógeno total (N) (Kjeldhal), pH en agua, Ca y Mg (acetato de amonio pH7 y absorción atómica), $\mathrm{K}$ y Na (acetato de amonio pH7 y emisión atómica).

\section{Análisis de datos}

Para el análisis del efecto de los tratamientos, las variables consideradas fueron: las propiedades físico-químicas del suelo y el número de individuos $/ \mathrm{m}^{2}$ total y de cada uno los 16 grupos taxonómicos evaluados.

Las propiedades físico-químicas son variables continuas que fueron analizadas utilizando modelos lineales generales (Proc. GLM, SAS Inst. 1999). La comparación entre tratamientos se realizó mediante la prueba de Mínima Diferencia Significativa (MDS).

El número de individuos $/ \mathrm{m}^{2}$ es una variable discreta que presentó una importante asociación entre la media y varianza de los tratamientos, razón por la cual fueron

Cuadro II. Clasificación taxonómica de los organismos integrantes de la macrofauna.

\begin{tabular}{llcl}
\hline \multicolumn{1}{c}{ Filo } & \multicolumn{1}{c}{ Clase } & Sub-Clase & \multicolumn{1}{c}{ Orden } \\
\hline Annelida & Clitellata & - & Oligochaeta \\
Arthropoda & Arachnida & - & Araneae \\
& Insecta & - & Coleoptera (larvas y adultos) \\
& & Dictyoptera \\
& & Diptera \\
& & Hemiptera \\
& & Hymenoptera \\
& & Homoptera \\
& & & Isoptera \\
& & & Lepidoptera \\
& & & Orthoptera \\
& & & Isopoda \\
& Crustacea & - & \\
& & & \\
Mematiapoda & Chilopoda & \\
Mollusca & Adenophorea & Diplopoda & \\
\hline
\end{tabular}


analizadas utilizando modelos lineales generalizados con distribución Poisson o binomial negativa y función logarítmica (Proc. GENMOD, SAS Inst. 1999).

Para explorar las relaciones entre las propiedades físico-químicas del suelo y la densidad de la macrofauna, en primer lugar se construyeron dos matrices con cada grupo de variables y como método de ordenación se utilizó el Análisis de Componentes Principales (ACP). Dado que las propiedades del suelo tienen distinta magnitud, los datos fueron estandarizados. Los grupos taxonómicos que estuvieron poco representados (menos del 1\% de la densidad) no fueron considerados. Posteriormente, los resultados de la ordenación de las propiedades del suelo y de la densidad de la macrofauna se conectaron mediante un análisis de Co-Inercia, que se utiliza como una técnica de ordenación que correlaciona los primeros ejes de ordenación de dos matrices. Este tipo de análisis de dos tablas trabaja en matriz de covarianza y permite explorar las relaciones cuando son consideradas muchas variables en pocos sitios de muestreo (Dolédec \& Chessel 1994). Para determinar la significación de los valores de Co-Inercia se realizó un test de Monte Carlo. El software utilizado fue ADE-4 (Thioulouse et al. 1997) incluido en el paquete R1.9 (R Development Core Team 2004).

Para determinar los grupos taxonómicos asociados a los distintos tratamientos se utilizó el Valor Indicador (IndVal) propuesto por Dufrêne y Legendre (1997). Este es un método simple que permite encontrar especies (o grupos taxonómicos) indicadoras de un grupo de muestras. Combina medidas del grado de especificidad de un taxon a un tipo de hábitat y su fidelidad dentro del mismo. Una fidelidad alta (frecuencia de ocurrencia) de la especie en los sitios de muestreo es generalmente asociada a una gran abundancia de individuos. Tiene un rango de 0 a 100 y alcanza el máximo cuando la especie está presente en todos los sitios que están relacionados y componen un grupo. Este método tiene ventajas sobre las otras medidas utilizadas, pues es calculado independientemente para cada taxon y no tiene restricciones en cuanto a la categorización de los sitios, que pueden ser agrupados arbitrariamente o para grupos de sitios determinados por algún método de clasificación (McGeoch \& Chow 1998). El cálculo del IndVal se realizó con el software PC-ORD 4.0 (McCune \& Mefford 1999).

\section{Propiedades físico-químicas del suelo}

El análisis de varianza de las propiedades del suelo evaluadas, determinó efecto de los tratamientos para C.Org., N, K, Mg, pH, y porcentaje de arcilla (Cuadro III). Se encontraron diferencias significativas entre las rotaciones agrícolas sin y con fertilización (S1 y S2) y las que incluyen leguminosas forrajeras en la rotación (S4, S5 y $\mathrm{S} 7)$ en el contenido de C.Org. $(\mathrm{P}<0.0001)$ y N $(\mathrm{P}<0.0001)$. En ambos casos, los menores valores registrados en los sistemas agrícolas respecto a los que consideran 
Cuadro III. Propiedades del suelo en los tratamientos del experimento de rotaciones cultivos-pasturas.

\begin{tabular}{lccccc}
\hline \multirow{2}{*}{ Propiedad } & \multicolumn{5}{c}{ Usos del suelo† } \\
\cline { 2 - 6 } & $\mathrm{S} 1$ & $\mathrm{~S} 2$ & $\mathrm{~S} 4$ & $\mathrm{~S} 5$ & $\mathrm{~S} 7$ \\
\hline Carbono Orgánico & $1.30 \mathrm{~d}^{*}$ & $1.62 \mathrm{c}$ & $2.53 \mathrm{a}$ & $2.36 \mathrm{ab}$ & $2.23 \mathrm{~b}$ \\
Nitrógeno Total & $0.13 \mathrm{~b}$ & $0.15 \mathrm{~b}$ & $0.23 \mathrm{a}$ & $0.22 \mathrm{a}$ & $0.22 \mathrm{a}$ \\
$\mathrm{Ca}$ & $11.47 \mathrm{a}$ & $10.57 \mathrm{a}$ & $12.56 \mathrm{a}$ & $11.36 \mathrm{a}$ & $12.28 \mathrm{a}$ \\
$\mathrm{Mg}$ & $3.71 \mathrm{a}$ & $2.80 \mathrm{c}$ & $3.61 \mathrm{a}$ & $2.87 \mathrm{bc}$ & $3.44 \mathrm{ab}$ \\
$\mathrm{K}$ & $0.82 \mathrm{bc}$ & $0.76 \mathrm{c}$ & $1.13 \mathrm{a}$ & $0.93 \mathrm{~b}$ & $1.13 \mathrm{a}$ \\
$\mathrm{Na}$ & $0.19 \mathrm{a}$ & $0.17 \mathrm{a}$ & $0.14 \mathrm{a}$ & $0.15 \mathrm{a}$ & $0.16 \mathrm{a}$ \\
$\mathrm{PH}$ & $6.11 \mathrm{a}$ & $5.83 \mathrm{~b}$ & $5.77 \mathrm{~b}$ & $5.73 \mathrm{~b}$ & $5.73 \mathrm{~b}$ \\
Arena (\%) & $20.67 \mathrm{a}$ & $21.00 \mathrm{a}$ & $19.67 \mathrm{a}$ & $20.00 \mathrm{a}$ & $19.67 \mathrm{a}$ \\
Limo (\%) & $47.67 \mathrm{a}$ & $49.00 \mathrm{a}$ & $48.33 \mathrm{a}$ & $49.33 \mathrm{a}$ & $45.33 \mathrm{a}$ \\
Arcilla (\%) & $31.67 \mathrm{ab}$ & $29.67 \mathrm{c}$ & $32.00 \mathrm{ab}$ & $30.67 \mathrm{~b}$ & $35.00 \mathrm{a}$ \\
\hline
\end{tabular}

*Valores seguidos por la misma letra no son significativamente diferentes $(\mathrm{P} \leq 0,05)$ para los contrastes de las medias de los usos de la tierra basado en la prueba de la Mínima Diferencia Significativa (MDS).

$\uparrow$ Para el significado de las abreviaciones de los usos del suelo, ver Cuadro I.

leguminosas forrajeras en la rotación son consecuencia básicamente de la erosión y del bajo ingreso de residuos orgánicos vegetales.

\section{Macrofauna del suelo}

En los dos muestreos fueron colectados un total de 3678 individuos; el $43 \%$ fue colectado en el muestreo de primavera (2004) y $56 \%$ en el muestro de otoño (2005). Los grupos taxonómicos más abundantes fueron Oligochaeta y Coleoptera inmaduros.

Las rotaciones de agricultura continua S1 y S2 tuvieron la menor riqueza, con 34 y 42 morfoespecies respectivamente. Por el contrario, en la rotación con período de pradera más prologado (S4) se computó la mayor riqueza total (85 morfoespecies). Los otros tratamientos con leguminosas forrajeras en la rotación (S5 y S7) tuvieron una posición intermedia entre S2 y S4, con respectivamente 65 y 69 morfotipos (Cuadro IV).

Los análisis estadísticos de máxima verosimilitud indican que hubo efecto significativo de los tratamientos para el número total de individuos por unidad de superficie $(\mathrm{P}<0.0001)$ y la densidad de los grupos taxonómicos Coleoptera adultos $(\mathrm{P}<$ $0.0001)$ e inmaduros $(\mathrm{P}<0.0001)$, Chilopoda $(\mathrm{P}<0.0001)$, Dictyoptera $(\mathrm{P}<0.0001)$, Hymenoptera $(\mathrm{P} \leq 0.0175)$, Nematoda Mermithidae $(\mathrm{P}<0.0001)$, Oligochaeta $(\mathrm{P}<$ $0.0001)$ y Orthoptera $(\mathrm{P}<0.0001)$ (Cuadro IV). Sólo en el caso de Nematoda Mermithidae se registró interacción tratamiento por mes de muestreo $(\mathrm{P} \leq 0.0453)$. 
Zerbino: Macrofauna del suelo en rotaciones cultivos-pasturas

Cuadro IV. Riqueza total y densidad (individuos $\mathrm{m}^{-2}$ ) total y de distintos grupos taxonómicos en los distintos tratamientos.

\begin{tabular}{lccccc}
\hline \multicolumn{1}{c}{ Parametro } & \multicolumn{5}{c}{ Usos del suelo $\dagger$} \\
\cline { 2 - 6 } & $\mathrm{S} 1$ & $\mathrm{~S} 2$ & $\mathrm{~S} 4$ & $\mathrm{~S} 5$ & $\mathrm{~S} 7$ \\
\hline Araneae & $0.18 \mathrm{a}$ & $0.18 \mathrm{a}$ & $2.84 \mathrm{a}$ & $0.36 \mathrm{a}$ & $1.42 \mathrm{a}$ \\
Coleoptera adultos & $1.96 \mathrm{~d}$ & $3.11 \mathrm{~cd}$ & $16.00 \mathrm{a}$ & $7.82 \mathrm{bc}$ & $12.62 \mathrm{ab}$ \\
Coleoptera inmaduros & $3.91 \mathrm{~d}$ & $24.89 \mathrm{c}$ & $87.47 \mathrm{a}$ & $102.93 \mathrm{ab}$ & $46.04 \mathrm{~b}$ \\
Chilopoda & $0.53 \mathrm{~b}$ & $0.53 \mathrm{~b}$ & $3.02 \mathrm{a}$ & $4.09 \mathrm{a}$ & $3.38 \mathrm{a}$ \\
Dictyoptera & $0.71 \mathrm{c}$ & $1.24 \mathrm{c}$ & $9.78 \mathrm{a}$ & $3.73 \mathrm{bc}$ & $6.93 \mathrm{ab}$ \\
Diplopoda & $0.00 \mathrm{a}$ & $0.00 \mathrm{a}$ & $0.00 \mathrm{a}$ & $0.18 \mathrm{a}$ & $0.18 \mathrm{a}$ \\
Diptera & $0.00 \mathrm{a}$ & $0.36 \mathrm{a}$ & $6.04 \mathrm{a}$ & $0.53 \mathrm{a}$ & $0.71 \mathrm{a}$ \\
Gastropoda & $0.00 \mathrm{a}$ & $0.00 \mathrm{a}$ & $0.18 \mathrm{a}$ & $0.18 \mathrm{a}$ & $0.00 \mathrm{a}$ \\
Hemiptera & $0.00 \mathrm{a}$ & $0.00 \mathrm{a}$ & $0.89 \mathrm{a}$ & $0.18 \mathrm{a}$ & $0.18 \mathrm{a}$ \\
Hymenoptera & $0.18 \mathrm{~b}$ & $0.18 \mathrm{~b}$ & $26.49 \mathrm{a}$ & $0.53 \mathrm{~b}$ & $1.07 \mathrm{a}$ \\
Isopoda & $0.36 \mathrm{a}$ & $0.18 \mathrm{a}$ & $4.27 \mathrm{a}$ & $1.42 \mathrm{a}$ & $4.62 \mathrm{a}$ \\
Isoptera & $0.00 \mathrm{a}$ & $0.00 \mathrm{a}$ & $0.00 \mathrm{a}$ & $0.36 \mathrm{a}$ & $0.36 \mathrm{a}$ \\
Lepidoptera & $0.18 \mathrm{a}$ & $0.00 \mathrm{a}$ & $0.71 \mathrm{a}$ & $0.18 \mathrm{a}$ & $0.53 \mathrm{a}$ \\
Oligochaeta & $1.60 \mathrm{~d}$ & $6.76 \mathrm{c}$ & $114.84 \mathrm{a}$ & $44.80 \mathrm{~b}$ & $52.27 \mathrm{~b}$ \\
Orthoptera & $0.36 \mathrm{c}$ & $1.42 \mathrm{bc}$ & $4.44 \mathrm{a}$ & $3.56 \mathrm{~b}$ & $1.24 \mathrm{c}$ \\
Nematoda Mermithidae & $1.78 \mathrm{c}$ & $3.02 \mathrm{bc}$ & $6.04 \mathrm{a}$ & $4.98 \mathrm{ab}$ & $7.29 \mathrm{a}$ \\
Densidad total & $11.73 \mathrm{~d} *$ & $41.87 \mathrm{c}$ & $283.02 \mathrm{a}$ & $175.82 \mathrm{~b}$ & $138.84 \mathrm{~b}$ \\
No. total de morfotipos & 34 & 42 & 85 & 65 & 69 \\
\hline
\end{tabular}

*Valores seguidos por la misma letra no son significativamente diferentes $(\mathrm{P} \leq 0,05)$ para los contrastes de las medias de los usos de la tierra basado en el estadístico de máxima verosimilitud.

$\dagger$ Para el significado de las abreviaciones de los usos del suelo, ver Cuadro I.

En todos los casos, las menores abundancias se registraron en los sistemas de rotaciones agrícolas S1 y S2. Estos dos tratamientos sólo fueron diferentes entre sí en la densidad de Coleoptera inmaduros y Oligochaeta.

Por el contrario, en el sistema de rotaciones cultivos-pasturas que permanece mayor tiempo bajo pasturas con leguminosas (S4) se obtuvo el mayor registro de individuos totales y de los grupos taxonómicos en los que hubo efecto del tratamiento. Este uso del suelo fue diferente de los restantes que consideran leguminosas forrajeras en la rotación (S5 y S7) en la densidad total de Oligochaeta y Orthoptera. Por otra parte fue similar a $\mathrm{S} 7$ para el número de individuos por unidad de superficie de los grupos taxonómicos Coleoptera adultos, Dictyoptera e Hymenoptera; mientras que en el caso de Coleoptera inmaduros fue semejante a S5.

Las rotaciones cultivos pasturas S5 y S7 tuvieron posiciones intermedias y similares entre sí para la densidad total y de todos los grupos taxonómicos, con excepción 
de los valores registrados para Hymenoptera y Orthoptera. La rotación S5 fue similar a la de agricultura continua S2 para la abundancia de Coleoptera adultos, Dictyoptera, Hymenoptera, Orthoptera y Nematoda Mermithidae. Por su parte S7 sólo fue similar a las rotaciones agrícolas en el número de Orthoptera por unidad de superficie.

\section{Relaciones entre propiedades del suelo y la macrofauna}

El análisis de CoInercia entre las variables ambientales y la densidad fue significativo $(\mathrm{P}=0,01)$, lo que indica que la co-estructura descrita por los dos primeros ejes de CoInercia fue próxima a las estructuras descritas en los análisis individuales de los dos grupos de variables. Los primeros ejes del análisis de CoInercia explicaron el 94\% y $5 \%$ de la estructura común compartida por las matrices de variables ambientales y de densidad, lo que enfatiza la importancia del primer eje, que separó a los usos del suelo de acuerdo a su intensidad. Los Oligochaeta, Coleoptera (inmaduros y adultos) y Dictyoptera estuvieron asociados a rotaciones de cultivos con pasturas, ambientes con mayor cantidad de C.Org., $\mathrm{N}$ y K, con $\mathrm{pH}$ más bajos, y contenidos de arena y $\mathrm{Na}$ menores que las rotaciones agrícolas (Fig. 1a, b, c). El segundo eje separó a los dos momentos de muestreo (Fig. 1a).

\section{Valor indicador (IndVal)}

De acuerdo al análisis del IndVal, los taxones Araneae, Coleoptera adultos, Hymenoptera, Orthoptera y Oligochaeta estuvieron asociados al uso del suelo menos intensivo (S4), mientras que Coleoptera inmaduros estuvieron asociados a S5 (Cuadro V).

\section{DISCUSIÓN}

La intensidad de uso del suelo fue el factor determinante en los resultados obtenidos en este experimento. Al considerar el número de cultivos por año, hubo un gradiente

Cuadro V. Valores Indicadores (IndVal) significativos $(\mathrm{P} \leq 0.05)$.

\begin{tabular}{lcccccc}
\hline Grupos taxonómicos & \multicolumn{3}{c}{ Usos del suelo† } & \multirow{2}{*}{$\begin{array}{c}\text { Test de Monte } \\
\text { Carlo P } \leq\end{array}$} \\
\cline { 2 - 5 } & S1 & S2 & S4 & S5 & S7 & \\
\hline Araneae & 1 & 1 & 48 & 1 & 19 & 0.0135 \\
Coleoptera adultos & 3 & 8 & 38 & 18 & 30 & 0.0165 \\
Coleoptera inmaduros & 1 & 9 & 33 & 39 & 17 & 0.0509 \\
Hymenoptera & 0 & 0 & 62 & 1 & 2 & 0.0465 \\
Oligochaeta & 0 & 3 & 52 & 20 & 24 & 0.0030 \\
Orthoptera & 1 & 9 & 40 & 27 & 8 & 0.0481 \\
\hline
\end{tabular}

†Para el significado de las abreviaciones de los usos del suelo, ver Cuadro I. 

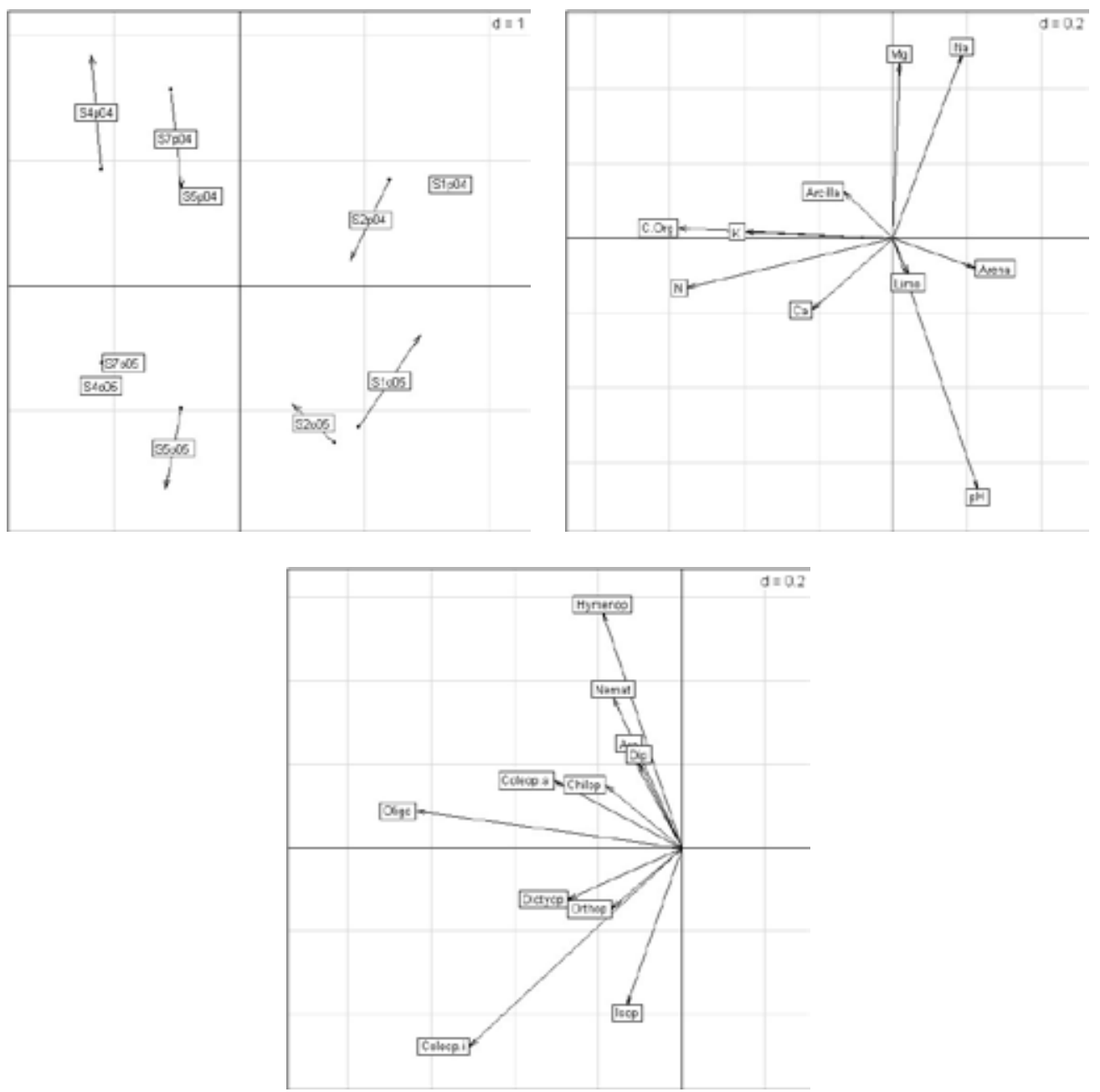

Figura 1. Análisis de Co-Inercia entre variables ambientales y número de morfoespecies: a) plano factorial de Co-Inercia de los usos de la tierra; b) y c) proyección de los vectores de las variables ambientales y de la riqueza de la macrofauna en el plano factorial de Co-Inercia . La punta de la flecha representa la posición del uso de la tierra para la biomasa de las morfoespecies y el otro extremo para las variables ambientales. Cuanto mayor es la flecha menor es la relación entre las variables ambientales y la biomasa de las morfoespecies. Abreviaciones de usos del suelo: S1-agricultura continua sin fertilización; S2-agricultura continua con fertilización; S4-rotación cultivos 33\%-pasturas 66\%; S5-rotación cultivos 50\%-pasturas 50\%; S7-rotación cultivos 66\%-pasturas 33\%. s04-setiembre 2004; a05-abril 2005. Abreviaciones del análisis del suelo: COrg-Carbono Orgánico; N-Nitrógeno Total; Ca-Calcio; Mg-Magnesio; K-Potasio; Na-Sodio. Abreviaciones de la fauna Del suelo: AraAraneae; Chilop-Chilopoda; Coleop.a-Coleoptera adultos; Coleop.I-Coleoptera inmaduros; DictyopDictyoptera; Dip-Diptera inmaduros; Hymenop-Hymenoptera; Isop-Isopoda; Nemat-Nematoda Mermithidae; Oligo-Oligochaeta; Orthop-Orthoptera. 
en la intensidad del uso del suelo desde los sistemas agrícolas S1 y S2 hasta el sistema de rotación S4 con mayor proporción de tiempo en pradera. Las diferencias en la proporción del tiempo en agricultura provoca cambios en la vegetación (estructura y densidad) y produce importantes variaciones en el microclima, en el hábitat y en la disponibilidad de recursos (Curry 1987a).

La riqueza y la densidad total de individuos y de los grupos taxonómicos en los que hubo efecto del tratamiento cambiaron según la frecuencia del disturbio físico y las diferencias en la vegetación. Los tratamientos que consideran leguminosas forrajeras en la rotación favorecieron la presencia de la macrofauna del suelo. La rotación S4 fue la que registró los valores más altos, señalando que este uso del suelo brindó las condiciones de hábitat más favorables, con mayor número de recursos a ser colonizados (Azevedo et al. 2000, Decäens et al. 2001).

A pesar de las diferencias en la intensidad de uso del suelo, los tratamientos S5 y S7 fueron semejantes en la abundancia de la mayoría de los taxones, sólo se diferenciando en las densidades de Hymenoptera y Orthoptera. Eso podría ser debido a las diferentes especies vegetales utilizadas en la etapa de pastura en cada rotación: en S5 se utiliza una mezcla de gramíneas y leguminosas mientras que en S7 se utiliza trébol rojo. Por lo tanto, las comunidades de la macrofauna del suelo estarían reflejando diferencias tanto en la vegetación cuanto en el manejo (Lavelle \& Spain 2001).

Por el contrario, los sistemas de rotaciones agrícolas S1 y S2 afectaron de manera negativa a la mayoría de los grupos taxonómicos, debido a su menor contenido de materia orgánica, decurrente de la degradación de la estructura del suelo por el laboreo convencional (Curry \& Good 1992, Brown et al. 2001). Además, la reducción de la cantidad y diversidad bioquímica de los exudados radiculares y hojarasca que ingresan al sistema, influye en la composición de las comunidades subterráneas provocando posiblemente una disminución en la tasa de descomposición de los restos vegetales presentes (Joshi et al. 2004). Estos tratamientos sólo fueron diferentes entre sí en la densidad total y la de los grupos taxonómicos Oligochaeta y Coleoptera inmaduros, lo que demuestra que el agregado de fertilizantes químicos no causa una mejora sustancial en las condiciones del hábitat y en la disponibilidad de recursos para la macrofauna en general, aunque para las lombrices de tierra endógeas eso podría ser importante (Edwards \& Lofty 1982).

Los grupos taxonómicos que mostraron ser más sensibles a los cambios en el uso del suelo fueron Coleoptera (adultos e inmaduros) y Oligochaeta. Estos fueron favorecidos por los tratamientos que tuvieron leguminosas forrajeras en la rotación. Las densidades de Coleoptera y Oligochaeta estuvieron relacionadas positivamente con el contenido C.Org. y N, tal como lo determinaron otros autores (Clapperton 2000, Fraser et al. 1994, Zerbino 2005).

La co-estructura descrita por los dos primeros ejes del análisis de Co-Inercia entre las variables ambientales fue próxima a las estructuras descritas en los análisis 
individuales de las especies y las variables edáficas, indicando que la macrofauna del suelo es capaz de reflejar los cambios en las condiciones del suelo en los diferentes sistemas de rotación. La posición de los tratamientos respondió a la intensidad del uso del suelo. En un extremo se ubicaron las rotaciones agrícolas S1 y S2 y en el otro la rotación $\mathrm{S} 4$, con la fase de pastura más larga. Los tratamientos S5 y S7 tuvieron una posición intermedia.

La composición de las comunidades de los distintos usos del suelo respondió a las diferencias en las propiedades del suelo. Los Oligochaeta, Coleoptera (inmaduros y adultos) y Dictyoptera estuvieron asociados a rotaciones de cultivos y pasturas, que fueron ambientes con mayor cantidad de C.Org., $\mathrm{N}$ y arcilla más elevados y menor contenido de arena y $\mathrm{Na}$ y $\mathrm{pH}$ más bajos que los sistemas de rotaciones agrícolas. Estos resultados concuerdan con Lavelle (1988), Lavelle et al. (1995) y Curry (1988) que señalaron que el contenido de materia orgánica, el estatus nutricional, el $\mathrm{pH}$ y la textura del suelo pueden determinar la composición y abundancia de las comunidades edáficas.

El análisis de IndVal determinó que cinco grupos taxonómicos estuvieron asociados a los distintos usos del suelo. Los taxones Araneae, Coleoptera adultos, Hymenoptera, Orthoptera y Oligochaeta estuvieron asociados al uso del suelo menos intensivo (S4), mientras que Coleoptera inmaduros estuvieron asociados a S5. Ninguno de ellos presentó valores de IndVal mayores de 70. De acuerdo a McGeoch et al. (2002) las unidades taxonómicas u especies con valores intermedios de especificidad, denominadas detectoras, pueden ser útiles para el monitoreo de cambios ambientales porque tienen diferentes grados de preferencia en los distintos hábitats. Por lo tanto las variaciones relativas de su abundancia en las distintas unidades analizadas pueden estar indicando la dirección en la que están ocurriendo los cambios. En este sentido, la riqueza y la densidad total de individuos y de los grupos taxonómicos Coleoptera adultos e inmaduros, Chilopoda, Dictyoptera, Hymenoptera, Nematoda Mermithidae, Oligochaeta y Orthoptera variaron de acuerdo a la frecuencia del disturbio físico, el número de cultivos por año, a la productividad de los usos del suelo considerados (cantidad y calidad de recursos) y a la capacidad de cada uno de ellos de adaptarse a los cambios. Los mayores y menores valores fueron registrados respectivamente en los sistemas menos y más perturbados y los grupos taxonómicos que discriminaron más los tratamientos fueron Coleoptera (adultos e inmaduros) y Oligochaeta.

Finalmente, la macrofauna del suelo estuvo relacionada con diferentes propiedades físicas y químicas del suelo, por lo que puede ser utilizada como indicadora. Los taxones Araneae, Coleoptera adultos, Hymenoptera, Orthoptera y Oligochaeta estuvieron asociados al uso del suelo con un período de pastura más prolongado; mientras que Coleoptera inmaduros estuvo asociado al tratamiento que tiene el 50\% del tiempo en fase pastura. 
Debido a la sensibilidad a las prácticas de manejo, la evaluación de las propiedades del suelo conjuntamente con la macrofauna que lo habita, puede ser una herramienta útil para evaluar la sustentabilidad ambiental de las prácticas de manejo de suelos y cultivos.

\section{AGRADECIMIENTOS}

A la Ing. Agr. $(\mathrm{PhD})$ Silvia Pereyra por la revisión de este trabajo, comentarios y sugerencias.

\section{LITERATURA CITADA}

Anderson, J. M. \& J. S. I. Ingram. 1993. Tropical soil biology and fertility. A handbook of methods, $2^{\text {nd }}$ Ed. CAB International, Wallingford.

Azevedo, V. F. de, D. A. Lima, M. E. F. Correia, A. M. Aquino \& H. P. dos Santos. 2000. Fauna de solo em diferentes sistemas de plantio e manejo no planalto medio do Rio Grande do Sul. In: Fertbio 2000. SBCS, Santa Maria. 1CD-Rom.

Brown, G. G., A. Pasini, N. P. Benito, A. M. Aquino \& M. E. F. Correia. 2001. Diversity and functional role of soil macrofauna communities in Brazilian no tillage agroecosystems: A preliminary analysis. In: International Symposium on Managing Biodiversity in Agricultural Ecosystems. FAO/ CBD, Montreal. 1CD-Rom.

Clapperton, J. 2000. Creating healthy productive soil. Pp. 35-40. In: VIII Congreso Nacional de AAPRESID, Mar del Plata.

Curry, J. P. 1987a. The invertebrate fauna of grassland and its influence on productivity. II. Factors affecting the abundance and composition of the fauna. Grass and Forage Science. 42: 197-212.

Curry, J. P. 1987b. The invertebrate fauna of grassland and its influence on productivity. III. Effects on soil fertility and plant growth. Grass and Forage Science. 42: 325-341.

Curry, J. P. 1988. Factors affecting earthworm abundance in soils. Pp. 37-64. In: C. A. Edwards (Ed.). Earthworm ecology. St Lucie Press, Boca Raton.

Curry, J. P. \& J. A. Good. 1992. Soil faunal degradation and restoration. Advances in Soil Science. 17: 171-215.

Decäens, T., P. Lavelle, J. J. Jiménez, G. Escobar, G. Rippstein, J. Schneidmadl, J. I. Sanz, P. Hoyos \& R. J. Thomas. 2001. Impact of land management on soil macrofauna in the eastern plains of Colombia. Pp. 19-41. In: J. J. Jiménez and R. J. Thomas (Eds.). Nature's plow: Soil macroinvertebrate communities in the Neotropical savannas of Colombia. CIAT Publication No. 324, Cali.

Dolédec, S. \& D. Chessel. 1994. Co-Inertia analysis: an alternative method for studying species-environment relationships. Freshwater Biology. 31: 277-294.

Doran, J. W. \& M. Safley. 1997. Defining and assessing soil health and sustainable productivity. Pp. 1-28. In: C. E. Pankhurst, B. M. Doube and V. V. S. R. Gupta (Eds.). Biological indicators of soil health. CAB International, Wallingford.

Dufrêne, M. \& P. Legendre. 1997. Species assemblages and indicator species: the need for a flexible asymmetrical approach. Ecological Monographs. 67: 345-366.

Edwards, C. A. \& J. R. Lofty. 1982. Nitrogenous fertilizers and earthworm populations in agricultural soils. Soil Biology \& Biochemistry. 14: 515-521.

Elliot, L. F. 1997. Soil biodiversity and grass cropping systems. Pp. 241-248. In: Proceedings of the $18^{\text {th }}$ International Grassland Congress. IGC, Winnipeg. 
Fraser, P. M., R. J. Haynes \& P. H. Williams. 1994. Effects of pasture improvement and intensive cultivation on microbial biomass, enzyme activities, and composition and size of earthworm populations. Biology and Fertlity Soils. 17: 185-190.

González, G., R. E. Ley, S. K. Schmidt, X. Zou \& T. R. Seastedt. 2001. Soil ecological interactions comparisons between tropical and subalpine forests. Oecologia. 128: 549-556.

Joshi, J., S. J. Otway, J. Koricheva, A. B. Pfisterer, J. Alphei, B. A. Roy, M. Scherer-Lorenzen, B. Schmid, E. Spehn \& A. Hector. 2004. Bottom-up effects and feedbacks in simple and diverse experimental grassland communities. Ecological Studies. 173: 115-134.

Lavelle, P. 1988. Earthworm activities and the soil system. Biology and Fertlity Soils. 6:237-251.

Lavelle, P. 2002. Functional domains in soils. Ecological Research. 17: 441-450.

Lavelle, P., E. Blanchart, A. Martin, S. Martin, I. Barois, F. Toutain, A. Spain \& R. Schaefer. 1993. A hierarchical model for decomposition in terrestrial ecosystems. Application to soils in the humid tropics. Biotropica. 25: 130-150.

Lavelle, P., A. Chauvel \& C. Fragoso. 1995. Faunal activity in acid soils. Pp. 201-211. In: R. A. Date (Ed.). Plant-soil interactions at low $\mathrm{pH}$. Kluwer, Netherlands.

Lavelle, P., T. Decaëns, M. Aubert, S. Barot, M. Blouin, F. Bureau, P. Margerie, P. Mora \& J. P. Rossi. 2006. Soil invertebrates and ecosystem services. European Journal of Soil Biology. 42: 3-15.

Lavelle, P. \& A. V. Spain. 2001. Soil ecology. Kluwer, Dordrecht.

Linden, D. R., P. F. Hendrix, D. C. Coleman \& P. C. J. van Vilet. 1994. Faunal indicators of soil quality. Pp. 91-106. In: J.W. Doran and A.J. Jones, A.J. (Eds.). Defining soil quality for a sustainable environment. SSSA Special Publication No. 35, Madison.

McGeoch, M. A. \& S.L. Chown. 1998. Scaling up the value of bioindicators. Trends in Ecology and Evolution. 13(2): 46-47.

McGeoch, M. A., B. J. Van Rensburg \& A. Botes. 2002. The verification and application of bioindicators: a case study of dung beetles in a savanna ecosystem. Journal of Applied Ecology. 39: 661-672.

McCune, B. \& M. J. Mefford. 1999. Multivariate analysis of ecological data. Version 4.0.

R Development Core Team 2004. R: A language and environment for statistical computing. R Foundation for Statistical Computing, Vienna.

SAS Institute Inc. 1999. SAS/STAT User's Guide, Version 8, Cary NC: SAS Institute Inc.

Thioulouse, J., D. Chessel, S. Dolédec \& J. M. Olivier. 1997. ADE-4: a multivariate analysis and graphical display software. Statistics and Computing. 7: 75-83.

Wardle, D. A. 1995. Impacts of disturbance on detritus food webs in agro-ecosystems of contrasting tillage and weed management practices. Advances in Ecological Research. 26: 105-185.

Zerbino, M. S. 2005. Evaluación de la densidad, biomasa y diversidad de la macrofauna del suelo en diferentes sistemas de producción. Tesis de Magister en Ciencias Ambientales, Universidad de la República, Facultad de Ciencias, Montevideo. 